cryptogenic West syndrome. J Pediatr March 2007;150:295-299). (Reprints: Dr Shin-ichiro Hamano, Division of Neurology, Saitama Children's Medical Center, 2100 Magome, Iwatsuki-ku, Saitama-city, Saitama 339-8551, Japan).

COMMENT. The importance of early diagnosis and treatment of infantile spasms, demonstrated by this study, confirms previous reports of a correlation of improved response and prompt initiation of ACTH therapy (Millichap JG, Bickford RG. JAMA 1962;182:523527). Of 21 patients referred to the Mayo Clinic, $80 \%$ of infants diagnosed and treated at $<1$ year of age were benefited whereas only $22 \%$ older than 1 year at diagnosis showed reduction in spasms and EEG improvement. The shorter the treatment lag, the more favorable the outcome. (Koo B et al. Neurology 1993;43:2322-7; cited by authors).

In the above study, patients with a poor outcome have a greater risk of focal epilepsy and persistent paroxysmal EEG discharges in frontal regions. In a long-term study of 214 patients followed for 20-35 years at the University of Helsinki, Finland (Riikonen R. Epilepsia 1996;37:367-372), factors predictive of a good prognosis included cryptogenic etiology, normal development before onset of spasms, and a good response to ACTH. Focal abnormalities in the EEG were not necessarily indicative of a poor prognosis.

\title{
ICTAL SPECT FOCAL HYPERPERFUSION IN WEST SYNDROME
}

Ictal single photon emission computed tomography (SPECT) and EEG were used to determine the mechanism of clustered spasms in 3 patients with symptomatic West syndrome (WS), in a study at Tokushima University, Japan. Regional cerebral blood flow increased during ictus and decreased during the interictal period in the area coinciding with the CT/MRI delineated focal cerebral lesion. Ictal hyperperfusion of bilateral basal ganglia was detected in 2 of 3 patients. Ictal EEG showed a diffuse slow wave complex corresponding to a clinical spasm. Sharp waves that preceded the delta activity and spasm were located in the same area in which cerebral blood flow increased during ictus. No patient showed a partial seizure. Focal cortical discharge and secondary generalization generate cluster spasms and trigger the brain stem and basal ganglia to produce spasms. Administration of clonazepam or resection of the focal cortical lesion resulted in complete cessation of spasms and disappearance of hypsarrythmia. (Mori K, Toda Y, Hashimoto T et al. Patients with West syndrome whose ictal SPECT showed focal cortical hyperperfusion. Brain Dev May 2007;29:202-209). (Respond: Dr Kenji Mori, Department of Pediatrics, School of Medicine, Tokushima University, Kuramoto-cho, Tokushima, Japan).

COMMENT. A previous SPECT study from Tokushima University (Miyasaki M et al. Epilepsia 1994;35:988-992; Ped Neur Briefs Dec 1994) showed localized cerebral hypoperfusion in the temporal lobes in 7 of 10 patients with infantile spasms, EEG showed corresponding focal abnormalities in 5, and the MRI confirmed localized lesions in only 3. PET studies have shown hypermetabolism of the lenticular nuclei in 32 of 44 infants with both cryptogenic and symptomatic infantile spasms. (Chugani HT et al. Ann Neurol 1992;31:212-219; Ped Neur Briefs March 1992). Every infant with a focal lesion on CT/MRI had a focal abnormality on PET in the same location, but 17 of 28 infants with focal abnormalities on PET had no detectable focal abnormalities on CT/MRI. Chugani HT et al 
also reported bitemporal hypometabolism on PET in 18 infants with spasms and severe developmental delay (Ann Neurol 1996;39:643-649).

\section{DEVELOPMENT OF WEST SYNDROME IN INFANTS WITH EPILEPTIC DISCHARGES}

Of 45 infants having epileptic discharges on the EEG in early infancy, 26 developed West syndrome (WS), in a study at Okayama University, Japan. Infants with periventricular leukomalacia (PVL) were more likely to develop WS than those without; they were also more likely to have WS than other types of epilepsy. Eleven out of 17 infants with PVL developed WS. Most infants with WS had developed initial epileptic discharges before 3 months of age. Preterm infants with PVL who show epileptic discharges on EEG before 3 months of age should receive antiepileptic treatment to prevent the onset of WS. (Yoshinaga $\mathrm{H}$, Endo F, Kikumoto $\mathrm{K}$ et al. Epilepsy development in infancy with epileptic discharges. Brain Dev May 2007;29:217-223). (Respond: Dr Harumi Yoshinaga, Department of Child Neurology, Okayama University Graduate School of Medicine, Japan).

COMMENT. A new model of flexion spasms involving prenatal exposure to betamethasone followed by injection of N-methyl-D-aspartic acid (NMDA) on day 15 postnatally in rats may provide a means of development and testing of new effective therapies for infantile spasms (Vellsek L et al. Ann Neurol Feb 2007;61:109-119; Baram TZ. Editorial Ann Neurol 61:89-91). These experimental spasms were sensitive to. ACTH therapy, ictal EEG electrodecremental or afterdischarges compatible with hypsarrhythmia were recorded, and imaging studies defined lesions in the limbic areas, hypothalamus, and brainstem associated with spasms.

\section{PROGNOSIS OF PARTIAL EPILEPSY PREDICTED BY MRI AND PET}

The evolution of cerebral glucose metabolism after partial seizure onset was studied in 38 children using PET scans over $3.0+/-1.3$ years (and within a year after a third unprovoked partial seizure) by researchers at the Clinical Epilepsy Section, NINDS, and Children's National Medical Center, Washington, DC. Twenty-eight patients with an initial normal PET were significantly more likely to have good seizure control than the 10 with initial abnormal scan. Those that developed an abnormal PET had longer history of epilepsy before the first PET but not greater seizure frequency. Patients with shorter time between seizures and higher seizure frequency had a greater risk of abnormal PET. Six of 7 patients with persistently abnormal PET had poor seizure control. History of febrile seizures did not affect PET findings. MRI was strongly predictive of initial PET results, but did not correlate with fluctuating hypometabolism. The combination of MRI and initial PET was strongly predictive of clinical course. (Gaillard WD, Weinstein S, Conry J et al. Prognosis of children with partial epilepsy. MRI and serial FDG-PET. Neurology February 2007;68:655-659). (Reprints: Dr William D Gaillard, Children's National Medical Center, 111Michigan Ave, NW, Washington, DC 20010).

COMMENT. Initial, but not serial, MRI and FDG-PET combined may be useful in predicting prognosis of partial epilepsy in children. 